\title{
MARITIME SURVEILLANCE USING SAR OCEANIC IMAGES
}

\author{
DR.J.Chenni Kumaran ${ }^{1}$, Dr.V.Suresh Kumar ${ }^{2}$, ThamaraiKannan $\mathrm{P}^{3}$, Manikandan $\mathrm{K}^{4}$, Isaiah prince $\mathrm{E}^{5}$ \\ ${ }^{1}$ Professor Department of Information Technology, Panimalar Institute of Technology, Chennai, INDIA \\ ${ }^{2}$ Professor and Principal, Adi Shankara Institute of Engineering and Technology, Ernakulam, INDIA \\ ${ }^{3,4,5}$ UG Scholar Department of Information Technology, Panimalar Institute of Technology, Chennai, INDIA \\ ${ }^{1}$ drchennikumaran@gmail.com, ${ }^{2}$ vsk72.suresh@gmail.com, ${ }^{3}$ pthamaraikannan007@ gmail.com, ${ }^{4}$ kamanikrish@gmail.com, ${ }^{5}$ \\ isiahprince67@gmail.com
}

\begin{abstract}
Marine things to do play a necessary position in the improvement of a nation. There is, however, a possible threat to the protection of a country via marine routes. This paper provides the most effective and easy-to-use method that enables the defense and marine protection authority staff to track the middle sections of the ocean for unusual marine activities by collecting an oceanic image from a SAR satellite. It helps the user by extracting information such as number of ships at particular region, distance between each, length and breadth of ships and the velocity at which the ship is moving. It includes various modules such as initial tiling and pre-processing, vessel or ship detection and wake detection for estimating velocity of ship. The interface of this framework is very user-friendly and fully implemented with a MATLAB.
\end{abstract}

Keywords: SAR, vessels, ships, wakes, filters, enhancement, tiling, satellite, radon, hough, kuan, kuwhara, frost, kelvin, turbulent, schimittles

\section{Introduction}

In recent years, maritime surveillance has been rapidly gaining advantages. Several satellites, such as RADARSAT-1, RADARSAT-2, ERS-1, ENVISAT, RISAT-1 and RISAT-2, have been dedicated to tracking the ocean and land surface from SAR images. A more comprehensive understanding of ocean surface features from satellite imagery is needed to promote effective and efficient management of maritime surveillance. Some ocean-related parameters are derived from SAR satellite images for the extraction of ocean features. The Synthetic Aperture Radar is satellite-attached imaging radar.

Electromagnetic waves are sequentially emitted, similar to traditional radar, and the radar antenna receives back scattered echoes. C-, X-, S-Band are mainly used for Agriculture, Ocean, Ice, land analysis and the $\mathrm{X}$-, Ku-Band are used for snow monitoring. Mostly used frequency bands are L-, C-, X- Bands.
The use of synthetic aperture radar (SAR) in Remote Sensing (RS) and Geographical Information Systems (GIS) is an important source of information for Earth observation [3]. In different applications, such as flood control, fire monitoring, ocean monitoring, epidemiological surveillance, etc., SAR images are being commonly used. SAR images, however, typically exhibit a speckle appearance that degrades SAR image quality [4]. Prior to moving to further research, it is desirable to reduce the speckle [10]. Latest SAR arrangements are capable $(\mathrm{HH} / \mathrm{HV} / \mathrm{VH} / \mathrm{VV})$ of providing fully polarized photos. Compared to single channel SAR, polarimetric SAR imagery has the advantage of having a comprehensive explanation of the scattering process of the land cover [8].

SAR images are analysed in this paper to extract ocean information, such as the number of ships in a specific area, the distance between them and their speed [4]. The overall hardware configuration of the SAR system is implemented on the satellite and how it collects a surface image using a transmitter and receiver is shown in Fig.1. Additional motion information is provided by the motion measurement sensor. Following signals from the analog to digital modulation block, the SAR processor block begins processing the SAR image. ERS-1, PALSAR, Sentinel satellite image in this document, which has requirements for capturing the ocean area and operating in strip MAP and SAR scan mode. The typical characteristic of SAR is that data can be collected at all times and under all-weather conditions. 


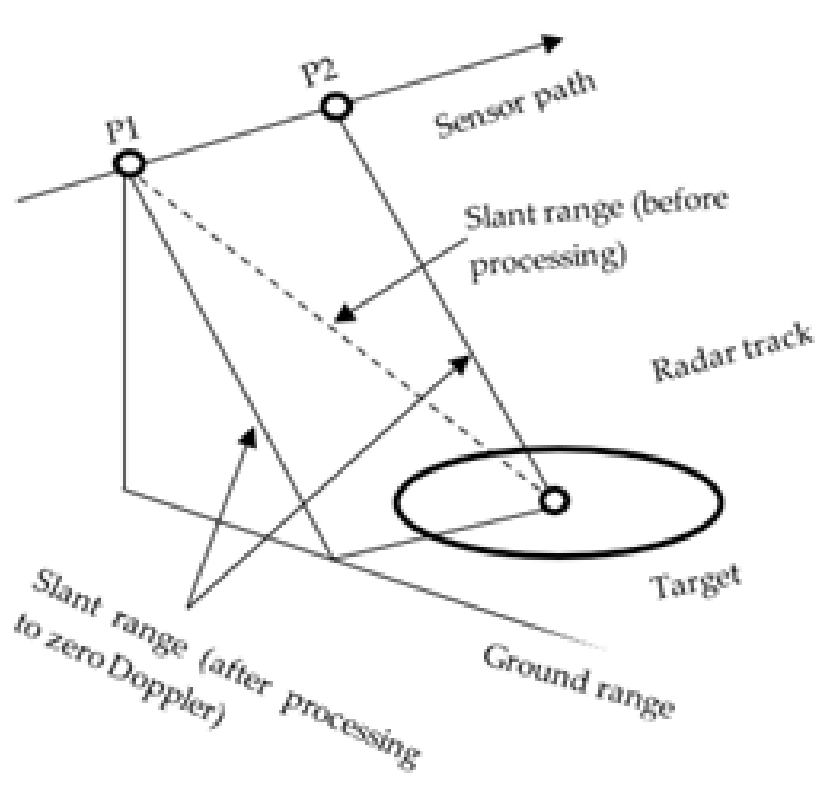

Fig.1 - Working of SAR

Its consistent image acquisition mechanism, however, introduces a lot of speckle noise in the SAR images, which makes the task very difficult, particularly finding the target region in the ocean to extract its characteristics [2]. With the laser imaging system, the SAR speckle system has a comparable principal [9]. The laser speckle characteristic is the same as the SAR speckle effect, which means that an unusual granular pattern in the picture is caused by the interference of coherent and reflected scatter. Despite the fact that dot holds itself data about enlightened region, it corrupts the presence of pictures and influences the exhibition of scene examination [7]. Therefore, the development of effective filtering techniques is definitely preferable, which can greatly decrease noise but at the same time retain entirely the appropriate details, such as ship and wake edges.

\section{Related Work}

Chen Yi, et al. [12] have built up a Radon change for distinguishing the direct surface in SAR picture. It is applied to programmed recognition of the boat wakes of SEASAT SAR picture. The area and course of the voyaging boat can be rapidly and precisely identified. Splendid or dim dashes of radar marks (back dispersing) can be applied to the recognition of moving boat. Data of the boat area, moving course, and surprisingly its speed can open up. One strategy is to initially distinguish the splendid focuses in SAR picture as potential areas of moving boats, and afterward they further identified the boat wakes around these focuses. In any case, it appears to be troublesome much of the time to confine the splendid focuses from fluctuating foundation of the SAR picture. Another technique proposed is to utilize a little square window moving over the picture to identify straight wakes.
F. Zhang et al. [13] have done transport discovery utilizing the versatile consistent bogus alert rate (CFAR) locator dependent on the chose likelihood thickness work. The location consequences of every district are combined. The examination depended on an ENVISAT ASAR picture. Boat signature in SAR pictures relies upon numerous components, for example, transport attributes, natural conditions, radar qualities, picture quality, picture goal, and so on The heterogeneities of SAR pictures brought about by advances between districts with various breeze conditions, current limits, breaking waves, low wind twisting imprints, impacts because of land regions like peripheral rocks, reefs and islands, backscattering modification because of bathymetry and the presence of boat wakes, oil spills, and so forth, present additional difficulties on transport recognition. These heterogeneities for the most part bring about an enormous number of bogus alerts when consistent bogus caution rate (CFAR) locators are applied.

Sudhir et.al [11] have utilized the prevailing frequencies recovered by methods for Quick Fourier Change (FFT) investigation regarding top recurrence reactions. Ocean surface slants were then gotten from the scattering connection with thought of various water wave conditions for every subset region, and wave statures were assessed with the utilization of predominant frequencies and ocean surface inclines. The technique is helpful to recover the different wave boundaries over various areas. Creators use RADARSAT-2 SAR sensor for sea wave estimation which works in the C-band $(5.4 \mathrm{GHz})$ of the electromagnetic range. SAR signals are more influenced by variety in the meteorological conditions, for example, ocean state, wind speed, and bearing. At last, they discover the frequency utilizing wave number.

\section{Proposed Work}

The purpose of the proposed work is primarily to extract ocean features from satellite imagery. The three significant operations used in SAR imagery to obtain ocean characteristics are presented. Fig. 2 displays the architecture of the future system. First is the pre-processing (image enhancement and filtering) of satellite imagery, the second is the retrieval of essential information from ocean satellite imagery such as vessel recognition, wake detection, vessel width, and the third is the calculation of vessel speed using a line detection algorithm. 


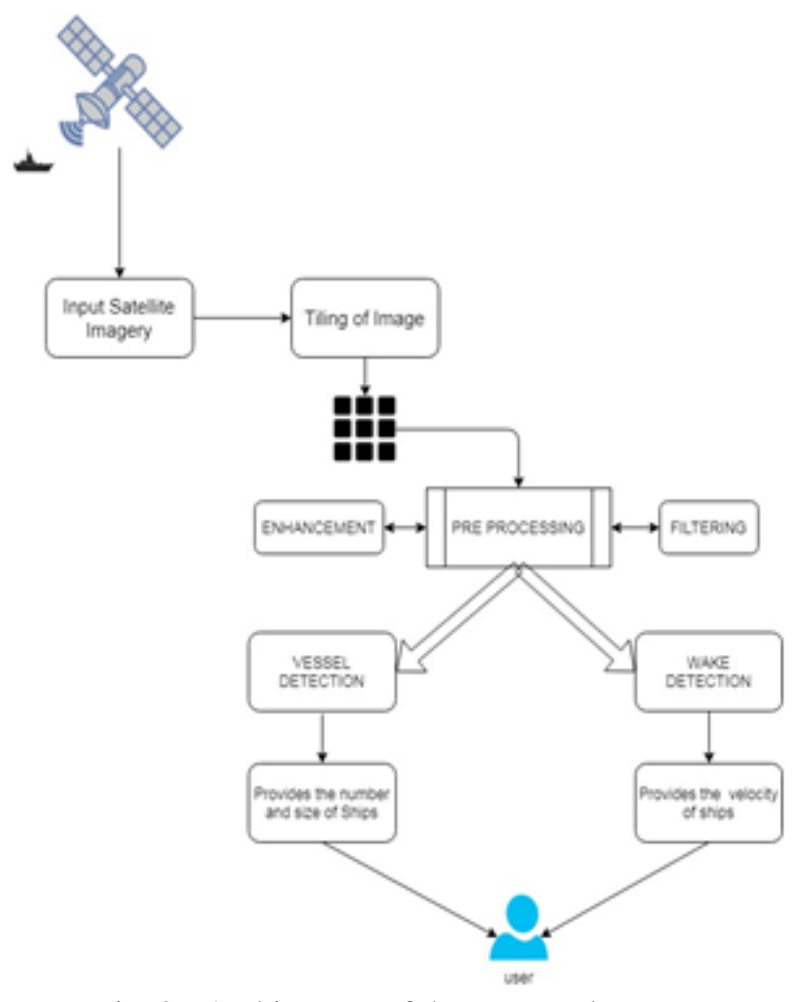

Fig. 2 - Architecture of the proposed system

\section{Methodology \& Results}

\subsection{Tiling and Pre-processing}

SAR image processing is especially time intensive and involves a high-performance computing device. To minimize the processing time, First add tiling to the image and then performed further analysis that involves speckle filtering and ship detection [1]. The time taken to apply filtering techniques and detection of ship takes around 20 minutes without tiling. Thanks to tiling, the rendering time for the SAR image is much shorter. Fig. 3 shows the Tiled Image. The tiling method is not needed for optical images since the non-linear filter is used to denounce the image. In general, the time consumption for nonlinear filtering is significantly smaller than for linear filtering. The pre-processing method can be categorized as an enhancement and filtering process.
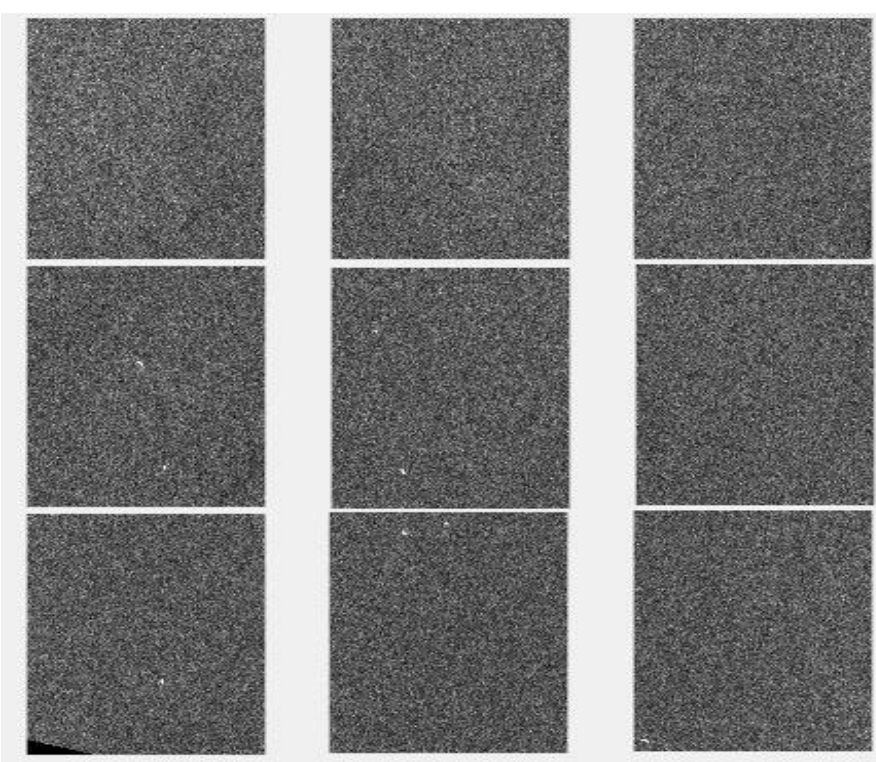

Fig. 3 - Tiled image

\subsubsection{SAR Image Enhancement Process}

Image enhancement is essentially enhancing the interpretability and interpretation of information in images for human audiences and provides improved feedback for other automatic image processing techniques. Image enhancement is meant to change the properties of an image to make it more suitable for a particular purpose and for a specific observer [6]. One or more attributes of the image are changed during this process. The choice of attributes and the manner in which they are changed are unique to a particular mission. Furthermore, observer-specific considerations, such as the human sensory system and the perspective of the observer, can add a great deal of subjectivity in the choice of imaging enhancement methods. There exist several strategies that can improve a digital image. Some of image enhancing approaches are Histogram Equalization, Adaptive Histogram Equalization. Fig. 4 shows the Enhanced Image Examples.

\section{Enhancement \& Filtering}
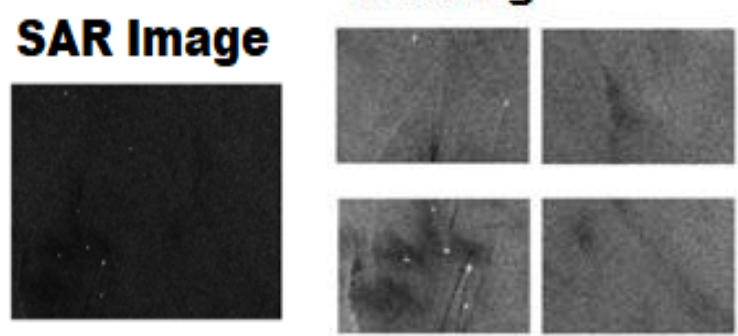


\section{Despeckled Image}

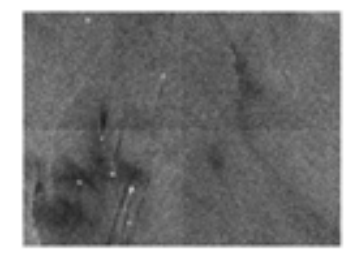

Fig. 4 Enhanced Image Examples

\subsubsection{SAR Image Filtering Process}

Similar linear and non-linear filters are used to eliminate noise from satellite imagery. The performance and ease of implementation are compared using different noise filters. The Edge Preservation Index (EPI) is also calculated for the purpose of understanding the preservation of the edge of the filtered images. The various filters applied here are median filter, hybrid median filter, lee filter, enhanced lee filter, kuan filter, frost filter and kuwhara filter.

\subsection{Vessel Detection}

By applying various morphological and statistical techniques, the number of vessels is detected precisely from the image. Once the vessel is detected, additional information such as length and width of the vessel, distance between multiple ships are extracted.

Using SAR Image, the number of ships in the ocean region is detected by using thresholding and morphological operations. It is useful for representation and outline of region shape.

The purpose of using morphological operations is to get rid of imperfections within the picture structure. Most of the operations used here are a mixture of two methods, dilatation and erosion [5]. After erosion and dilation, bound the ship using the bounding box form of the area props. Fig. 5 shows the Detection of Ships from SAR image.

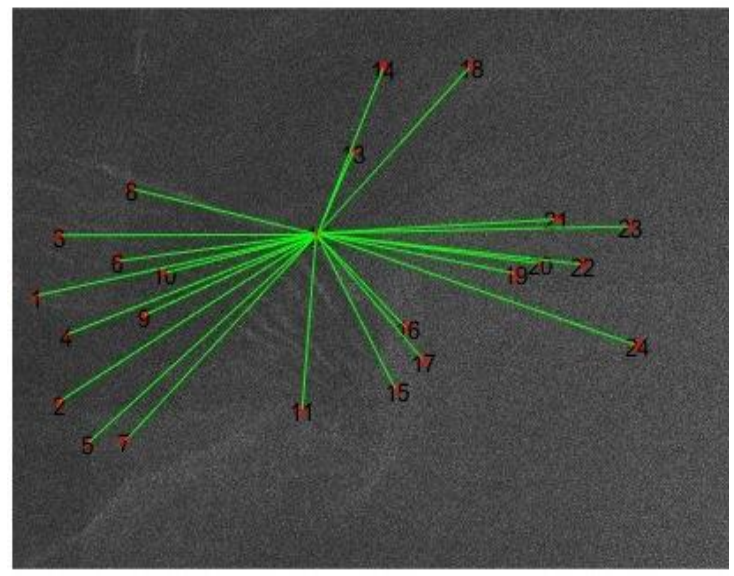

Fig. 5 - Detection of Ships from SAR image

\section{Opening: Erosion and then Dilation \\ $A$ o $B=(A \oplus B) \oplus B$}

Closing: Dilation and then Erosion

\section{$\boldsymbol{A} \cdot \boldsymbol{B}=(\boldsymbol{A} \oplus \boldsymbol{B}) \boldsymbol{\theta} \boldsymbol{B}$}

Dilation: The yield pixel is determined as the limit of the multitude of pixels in the neighbourhoods of the information pixel. In the picture, if any of the pixels is set to the worth 1, the yield pixel is set to 1 . Filling of holes of certain shape and size is given by the structural element.

Erosion: The yield pixel is determined as the base of the relative multitude of pixels in the neighbourhoods of the info pixel. In the picture, if any of the pixels is set to 0 , the yield pixel is set to 0 . Removal of structures of certain shape and size is given by the structural element.

After identifying ships, measuring the total number of ships present in the SAR picture and finding the distance between a single ship and another chosen ship or evaluating the distance between one and more ships, the distance is measured in terms of nautical miles. The length and breadth of the ships was therefore measured.

\subsection{Wake detection}

Wake is the pattern left by any object that is moving at or near the water surface. It forms around and immediately behind the ship and appears very dynamic, consisting of various components, such as bow and stern waves, but also eddies, tides and foam. The wake contour depends on a variety of factors, such as the actual structure of the ship, its height, wind strength and direction, and a number of other elements, but the length of the ship behind the ship becomes very universal.

The wake lines are detected using line detection algorithms they are 1. Radon Transform 2. Hough Transform.

Radon transform is useful for finding of lines in SAR pictures. It is based on the convolution of the image with a kernel that gives high values at output pixels where a line is present, and low values where the line tendency is low. Preferably the picture has been pre-processed with an edge enhancing filter to reduce noise the affecting the output.

Applying the Radon change on a picture $f(x, y)$ for a given arrangement of points can be considered as processing the projection of the picture along the given points. The subsequent projection is the amount of the powers of the pixels toward every path, for example a line necessary. The outcome is another picture $\mathrm{R}(\rho, \theta)$. This can be composed numerically as $\rho=x \cos \theta+y \sin \theta$

By using radon transform, one can detects the maximum peak values from the input image. From that maximum peaks, draw the line in the input image. The Radon change is broadly used to distinguish transport wakes in engineered gap radar (SAR) pictures. Boat wakes have straight highlights in the picture, and they compare to tops in the Radon change.

Hence, the boat wakes can be distinguished via looking for tops in the Radon change. Likewise, detect the ship wakes edge line using radon maximum peaks and radon lines. Fig. 6 shows the working of radon transform. 

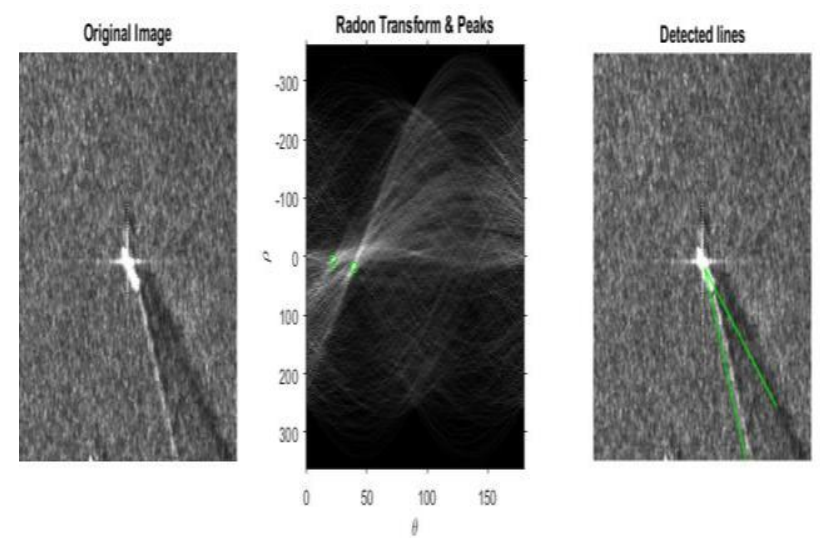

Fig. 6 - Working of Radon Transform

The Hough transform is an element extraction procedure utilized in picture investigation, PC vision, and advanced picture handling. The motivation behind the method is to discover blemished occurrences of articles inside a specific class of shapes by a democratic strategy. This democratic system is completed in a boundary space, from which article competitors are acquired as neighbourhoods maxima in a supposed collector space that is unequivocally built by the calculation for registering the Hough transform.

$r=x \cos \theta+y \sin \theta$

$y=-\frac{\cos \theta}{\sin \theta} \cdot x+\frac{r}{\sin \theta}$

Hough transform is a line detection technique used in satellite image processing. It takes a binary edge map as info and endeavours to find.

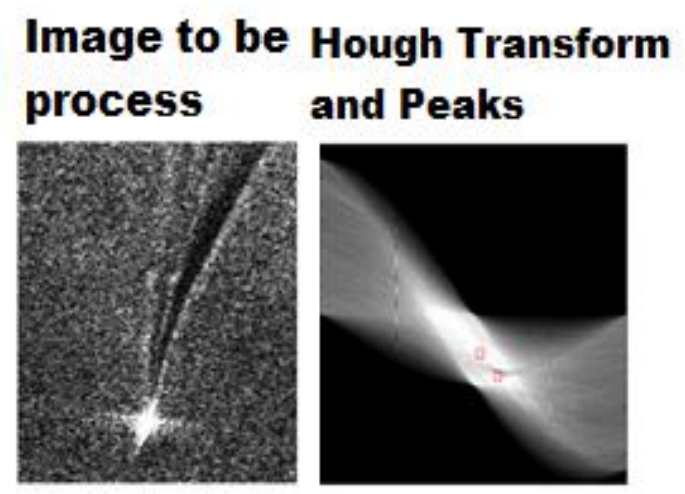

Wake Detection

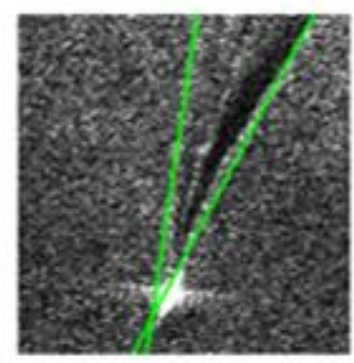

Fig. 7 - Hough Transform
The possibility of the Hough transform is, that each edge point in the edge map is changed to all potential lines that could go through that point. Each edge point is transformed to a line in the Hough space, and the aftereffect of the Hough transform is put away in a grid that is frequently called as an aggregator. One component of this lattice is the points $\theta$ and the other measurement is the distances $r$, and every component has a worth telling the number of focuses or pixels are situated on the line with boundaries $(r, \theta)$. Thus, the component with the most noteworthy worth determines what line that is generally addressed in the info picture. Fig. 7 shows the working of Hough transform. The parameters obtained from the following algorithm are used to estimate the velocity of the moving target in synthetic aperture radar imaging.

For our purpose of measuring ship speed, the main element of the wake design is that it moves along with the boat. Along these lines, there is a basic connection between the boat speed $\mathrm{v}$, the wave speed $\mathrm{c}$ and the point $\theta$ that the wave front makes with the boat's way the areas where most Hough space lines intersect is interpreted as true lines in the edge map. The displacement of the wave in the direction perpendicular to the front is $\mathrm{c}$ (c being the phase velocity). On deep water, surface waves of wavelength $\lambda$ have phase velocity. the velocity is estimated using the formula:

$$
v=\sqrt{\frac{g \lambda}{2 \pi}} \frac{1}{\sin \theta}
$$

\section{Conclusion}

This paper focuses on the information exploitation from the satellite imagery Synthetic Aperture Radar (SAR) of ocean regions. Since the speckle in SAR images degrade the quality of images, we used suitable filters to reduce the noise and also preserving important information such as wakes and edges [7]. In this paper three modules are implemented: Pre-processing, Vessel detection and Wake detection. In pre-processing we have applied different picture upgrade and sifting strategies to improve the nature of the picture prior to vessel and wake detection [6]. To reduce the processing time, we have used tiling approach, i.e., we split the image into appropriate number of tiles and process them individually and finally combine them into a single image. The second module in this project is Vessel Detection model, using morphological operations we were able to detect the vessels from the filtered images and also bounded the vessel area using relevant bounding boxes [2]. Finally, wake detection method was implemented using two-line detection algorithms namely Radon transform and Hough transform to estimate velocity of ship. The outcomes of this software are tested and verified using a SNAP toolbox. 


\section{References}

[1] Philippe Salembier ,Sergi Liesegang, and Carlos López Martínez, Senior Members, IEEE. "Ship Detection in SAR Images Based on Max tree Representation and Graph Signal Processing”, IEEE transactions on remote sensing, Vol. 57, No.5, pp. 2709-2724, MAY 2019.

[2] Weibo Huo, Yulin Huang, Jifang Pei, QianZhang, QinGuand Jianyu Yang." Ship Detection from Ocean SAR Image Based on Local Contrast Variance Weighted Information Entropy" IEEE, 2018 Apr; 18(4): 1196.

[3] Alberto Moreira, Pau Prats-Iraola, "A tutorial on Synthetic Aperture Radar", IEEE Geoscience and Remote Sensing Magazine, Vol.1, No.1, pp.6-43, April 2013.

[4] C. Wang, F. Bi, W. Zhang, and L. Chen, "An intensity-space domain CFAR method for ship detection in HR SAR images," IEEE Geosci. Remote Sens. Lett., vol. 14, no. 4, pp. 529-533, Apr. 2017.

[5] Jeya Ramya, V, Navarajan, J, Prathipa, R, Ashok Kumar, L, "Detection of melanoma skin cancer using digital camera images", ARPN Journal of Engineering and Applied Sciences, Vol. 10, No. 7, pp. 3082-3085, 2015.

[6] MA Jing, ZOU Chengming, JIN Xiaolong, “An Improved Image Enhancement Algorithm”, Journal of Natural Science, Vol.22 No.1, 085-092, 2017.

[7] Sahel mahdavi, Bahram Salehi, Cecilia Moloney, "Speckle Filtering of Synthetic Aperture Radar Images using Filter with Object-size-adapted window", International Journal of Digital earth, 2017.

[8] Fengkai Lang, Jie Yang and Deren Li, "AdaptiveWindow Polarimetric SAR Image Speckle Filtering Based on a Homogeneity Measurement", IEEE Transaction on geoscience and remote sensing, Vol.53, No.10, pp. 5435 - 5446, 2015.

[9] Y. Dong, A. K. Milne and B. C. Forster, "Towards Edge Sharpening- A SAR Speckle Filtering Algorithm", IEEE Transaction on geoscience and remote sensing, Vol, 39, No.4, pp. 851 - 863, 2001.

[10] Samuel Foucher, Carlos, “Analysis, Evaluation, and Comparison of Polarimetric SAR Speckle Filtering Techniques", IEEE Transactions On Image Processing, Vol. 23, No. 4, pp. 1751-1764, April 2014.

[11] Sudhir, Palanisamy, Chan-Su Yang and Ugur Guven, "Detection of Ocean Wave Parameters Using Synthetic Aperture Radar (SAR) Data", The
Journal Of Navigation, 66, No.2, 283-293, March 2013.

[12] Chen Yi, Jin Yaqui, "Detection of Ship Wakes in SAR Image using Rotated Window radon Transfrom", JOURNAL OF ELECTRONICS , Vol.19, 2002.

[13] F. Zhang and B. Wu," A scheme for ship detection in inhomogeneous regions based on segmentation of SAR images", International Journal of Remote Sensing Vol. 29, No. 19, 5733. 